\title{
Evaluation of the Association Between Matrix Metalloproteinase 11 and Intervertebral Disc Disease
}

\author{
Adem Bozkurt ARAS ${ }^{1}$, Mustafa GUVEN ${ }^{1}$, Naci BALAK², Erdoğan AYAN³ ${ }^{3}$, Suheyla BOZKURT UYAR ${ }^{4}$, Ilhan ELMACl ${ }^{5}$ \\ ${ }^{1}$ Canakkale Onsekiz Mart University, School of Medicine, Department of Neurosurgery, Canakkale, Turkey \\ ${ }^{2}$ Göztepe Education and Research Hospital, Department of Neurosurgery, Istanbul, Turkey \\ ${ }^{3}$ Trakya University, School of Medicine, Department of Neurosurgery, Edirne, Turkey \\ ${ }^{4}$ Marmara University, Institute of Neurological Sciences, Department of Pathology, Istanbul, Turkey \\ ${ }^{5}$ Medipol University, School of Medicine, Department of Neurosurgery, Istanbul, Turkey
}

\section{ABSTRACT}

\begin{abstract}
AIM: The intervertebral disc starts to degenerate when a human being begins to stand and learn to walk. It is known that many extrinsic, intrinsic and genetic factors play a role in disc degeneration. In this study, we examined whether the matrix metalloproteinase 11 might be associated with intervertebral disc degeneration.
\end{abstract}

MATERIAL and METHODS: Fifty-six patients with lumbar disc herniations who were operated at Göztepe Education and Research Hospital, Neurosurgery Clinic between September 2008 and December 2009 were prospectively reviewed. History and complaints were obtained from the case reports. Neuroradiological evaluation was performed with magnetic resonance imaging. Surgical findings of cases were reported in the operation notes. Microscopic posterior hemipartial laminectomy and discectomy were performed in all cases. Degenerated herniated disc material of all cases extracted during surgery was evaluated with immunohistochemical staining in Marmara University, Institute of Neurological Sciences, Pathology Laboratory.

RESULTS: Comparing the immunohistochemical staining of cases who were 50 years or younger and cases who were over 50 years old, statistical significance was determined.

CONCLUSION: Matrix metalloproteinase 11 has a role in degenerating intervertebral disc disease, but it is not the only factor. Matrix metalloproteinase 11 might be a genetic factor in young-middle aged patients.

KEYWORDS: Intervertebral disc degeneration, Intervertebral disc displacement, Matrix metalloproteinase 11, Middle age

\section{INTRODUCTION}

Intervertebral discs start to degenerate from the moment a human begins to stand and walk $(3,8)$. Degeneration of discs is not a result solely of aging and misuse of the lower back. It is known that many extrinsic, intrinsic and genetic factors play a role in the degeneration of discs (15). Generally, the effect of extrinsic factors such as trauma, heavy load lifting and working conditions on degeneration has been addressed (14). Systemic diseases (14), smoking (5), as well as genetic factors, can also degenerate the disc $(3,12)$. For this reason, the structure of intervertebral discs and pathophysiology of degeneration should be well understood in parallel to the development of molecular medicine. The prolongation of human life results in formation of degeneration of intervertebral disc and disc herniation with age, and therefore, research into new treatment choices is necessary.

The extracellular matrix structure of intervertebral discs consists of collagens, proteoglycans and non-collagen proteins. The biological basis of healthy disc tissue is based on disc cells functioning properly. The intervertebral disc is a tissue that is poor in term of cells. Cells of the disc consist of fibrocytes and chondrocytes. Cells occupy $1-5 \%$ of disc 
volume. The number of cells diminishes rapidly from the cartilage end plates towards the nucleus. End plate cells are typical chondrocytes $(10,19)$.

Matrix metalloproteinase (MMP) is released in inactive proenzyme form in the extracellular matrix. It is considered to play a role in disc degeneration by degrading collagen and proteoglycans found in the matrix $(1,13)$. The role of biochemical mediators seen in the degeneration of discs has not attracted much attention until recently. In recent years, metalloproteinases have come to the forefront among related proteinases after the existence of proteolytic enzymes in the disc structure was discovered. Many studies have concluded that expressions of enzymes that degrade the matrix in intervertebral disc degeneration increase. It was shown in the literature that some alleles of the MMP-3 gene are associated with disc degeneration of elderly Japanese patients (2).

MMP-11, from the metalloproteinase family, is a proteinase weighing $47 \mathrm{kDA}$. It is differentiated from other MMP members in terms of both enzymatic activity and activation. It has an extremely wide substrate range; it has features of splitting the non-helical region of proteoglycan, laminin, fibronectin casein and collagen (7). It was shown in the literature that other stromelysin group enzymes have increased expression in osteoarthritis patients and also MMP-11 enzyme activity increased in the late stage of the same patients (6). There is no data in the literature regarding a role for it in disc degeneration. However, many studies have shown other MMP group enzymes playing such a role (11).

\section{MATERIAL and METHODS}

Fifty-six consecutive patients with herniated lumbar disc who were operated on between September 2008 and December 2009 were analyzed prospectively. The study had the approval of an ethics committee. Histories and complaints of the cases were obtained from their records. Neuroradiological assessment of the cases was performed with magnetic resonance imaging (MRI) work-up. Schneiderman Disc Degeneration classification was applied in T2-weighted MRI scans during the neuroradiological assessment, as described previously (22) (Table I). Possible correlations between variables such as MMP-11 staining score, Schneiderman score, surgery level, gender and age group were investigated.

\section{Surgical and Histopathological Classifications}

Surgical findings of the cases were determined by examination of the operation notes of the cases. Standard microscopic hemipartial laminectomy, foraminotomy and discectomy were applied in all cases. Samples obtained from the herniated and degenerated disc material during the surgery were examined histopathologically. Specimens were kept in $10 \%$ formalin at room temperature for 24 hours and then they were embedded into paraffin. Four $\mathrm{mm}$ histological sections were prepared from paraffin blocks on lams coated with 3-amino propyl-triethoxysilane (A-3648, Sigma Aldrich Inc., USA). Degenerated intervertebral disc tissue specimens underwent immunohistochemical processes and were stained with ready-to-use monoclonal antibodies. The monoclonal antibody used, MMP-11 mouse monoclonal antibody, was provided by Santa Cruz Inc. (SC MS-1035-R7).

Prior to immunohistochemical analysis, control tissues envisaged for each antibody were stained. After positive staining was determined, a test group was stained and evaluated.

Immunohistochemical density evaluation was performed by the neuropathologist according to a semi-quantitative analysis system. Classifications were no staining (-), focal staining (staining below 10\%) (+), moderate staining (staining between $10-50 \%)(++)$, and prevalent staining (staining above $50 \%$ ) $(+++)$ (Table II).

\section{RESULTS}

Age and gender data, staining score and MRI score belonging to operations of 56 cases that were the subject of the research and these operation levels were examined. After descriptive statistics of variables were examined with statistical analysis, Chi-Square analysis was applied. Analysis was conducted using SPSS 16.0 (SPSS Inc., Chicago, IL, USA) software and an error margin of $5 \%$ was accepted.

Fifty percent of the cases consisted of women and $50 \%$ were men. Ages of cases ranged between 13 and 77 years. Average age was 47. Cases were divided into two groups as above 50 years old and below 50 years old. There were 30 cases in the $\leq 50$ years group and 26 cases above 50 years old.

Staining was not observed in $57.1 \%$ of cases (32 patients), staining less than $10 \%$ was seen in $28.6 \%$ (16 patients), $10-50 \%$ staining in $10.7 \%$ (6 patients), and more than $50 \%$ staining was found in 3.6\% (2 patients) (Figure 1A-D).

Grade I degeneration was found in $3.6 \%$ of cases (2 patients), Grade II in 62.5\% (35 patients) and Grade III in 33.9\% (19 patients) in evaluation of MRI findings (Figure 2A-D).

Operations were performed at L3-L4 level in $10.7 \%$ of cases (6 patients), at L4-L5 level in 58.9\% (33 patients) and L5-S1 level in $30.4 \%$ (17 patients).

While the MMP-11 staining in the group 50 years and younger was $56 \%$, this rate was $26.9 \%$ in the group above 50 years of age. Statistical significance was determined between staining degrees of patients aged $\leq 50$ and staining degrees of patients aged $>50$. This implies that the MMP-11 enzyme may play a role in disc degeneration in patient aged 50 years and younger. MMP-11 positivity was seen in $42.3 \%$, MMP-11 negativity in $57.1 \%$ in this study.

Table I: Schneiderman Classification of Disc Degeneration

\begin{tabular}{ll}
\hline Grade 0 & Normal (Hyperintense nucleus pulposus signal) \\
\hline Grade I & Slight reduction in nucleus pulposus signal \\
\hline Grade II & Global reduction in nucleus pulposus signal \\
\hline Grade III & $\begin{array}{l}\text { Hypointense nucleus pulposus signal and disc } \\
\text { space narrowing }\end{array}$ \\
\hline
\end{tabular}


There was no statistically significant correlation observed between the age groups and Schneiderman core, between age groups and surgery level, between gender and Schneiderman score, between gender and surgery level, between gender and staining scores, between Schneiderman score and staining score, between surgery level and staining score and between Schneiderman score and surgery level $(p<0.05)$.

\section{DISCUSSION}

Matrix metalloproteinases are proteinases found in the extracellular matrix structure. The human MMP family has 27 members. The MMP enzyme family is proteolytic enzymes. Essentially, they play a role in the destruction of protein. It undertakes several roles in growth, differentiation, apoptosis of cells, migration of cells, invasion and angiogenesis in tumors. MMP is released in an inactive proenzyme form in the extracellular matrix. MMP's are active in intervertebral disc matrices and degrade collagen and proteoglycans found in the matrix. Matrix metalloproteinases with the broadest activity area (MMP-3,10,11) degrade big matrix proteoglycans, nucleus protein, laminin, fibronectin, gelatin, and Type II, III, IV, V, IX collagens $(11,20)$.

Table II: Immunohistochemical Staining Scores of the Patients

\begin{tabular}{lcc}
\hline Staining score & Number of patients (n) & Percent (\%) \\
\hline No staining (-) & 32 & 57.1 \\
\hline Focal staining (staining below 10\%) (+) & 16 & 28.6 \\
\hline Moderate staining (staining 10-50\%) (++) & 6 & 10.7 \\
\hline Prevalent staining (staining above 50\%) (+++) & 2 & 3.6 \\
\hline Total & 56 & 100 \\
\hline
\end{tabular}

A

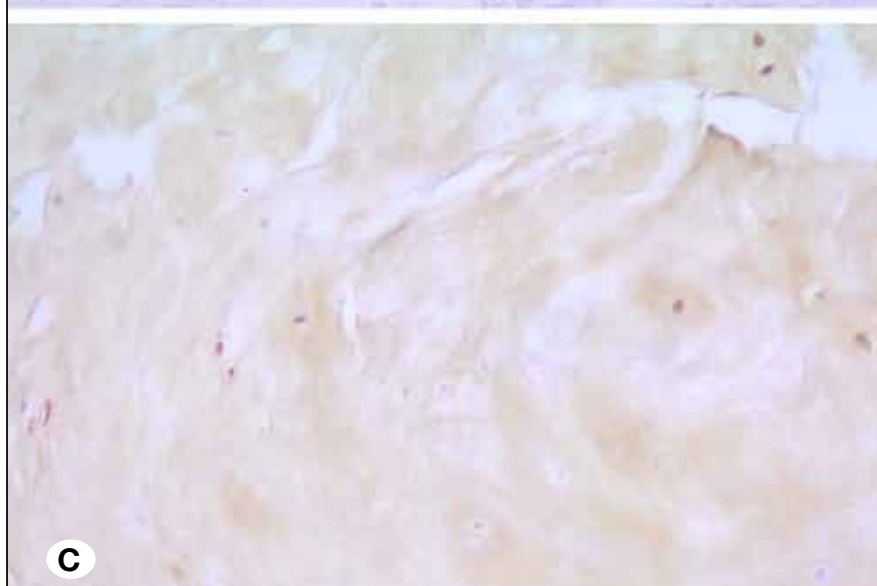

B

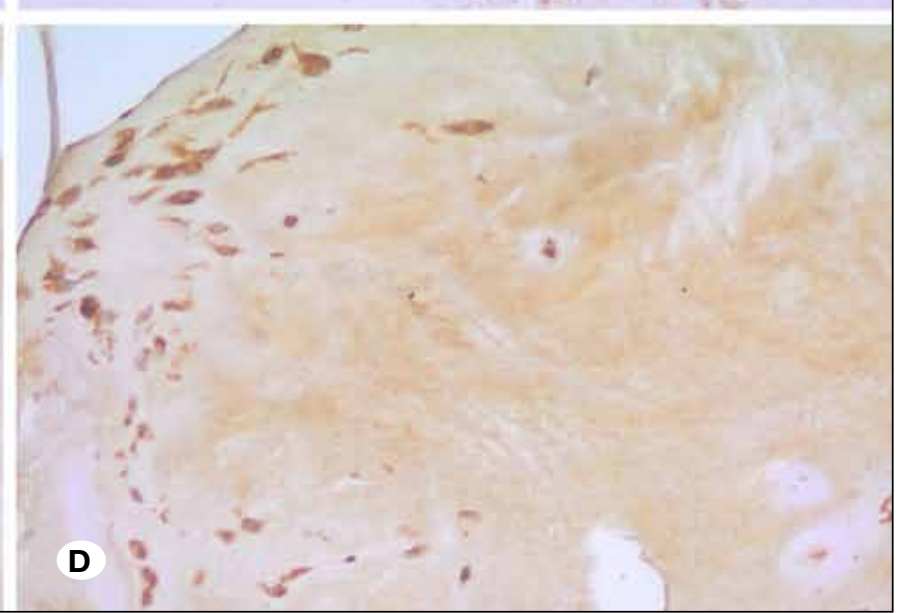

Figure 1: MMP-11 immunohistochemical staining of disc cartilage showed A) no immunohistochemical staining, B) focal immunohistochemical staining (staining below 10\%), C) moderate immunohistochemical staining (staining between 10-50\%), and D) prevalent immunohistochemical staining (staining above $50 \%$ ) (magnification: $x 20$ ). 
Risk factors in intervertebral disc herniation have been determined as young-middle age, male gender, familial tendency, environmental factors, trauma and smoking. In a broad study conducted, the female/male ratio was $1 / 1.6$. The percentages were equal in this study. Degeneration in discs below L4 level develops more quickly due to bio-mechanics and the physical weight of the spine, and this allows the formation of disc hernia (4). This study is in accord with that statement.

Miller stated that intervertebral discs undergo one of the most dramatic changes among connective tissues in the human body (17). There are many studies showing that degeneration increases and neuroradiological findings deteriorate with aging (16). In our study, it was seen that the degeneration degree increases as age increases when degeneration findings were evaluated by age and MRI scans.

Many studies show that expressions of enzymes degrading the matrix increase in intervertebral disc degeneration (21). These are MMP and ADAMS TS enzyme families. The most important enzyme group responsible for degradation of proteoglycans found in the structure of a disc is MMP (21). There are many studies concluding that the stromelysin group of enzymes plays a role in disc degeneration.

In a study conducted by Barksby et al., MMP-10 expression was isolated from hip cartilage chondrocytes in patients with osteoarthritis. The same study showed that MMP-10 enzyme activated pro MMP-1, 8, 11, 13 (4).

In a study by Richardson et al., the MMP-10 enzyme's role in disc degeneration was investigated. It was found that MMP10 enzyme expression is significantly high in degenerated disc specimens. Substantially dense staining was seen in immunohistochemical staining specimens (21). This increase in MMP-10 enzyme leads us to suggest that they have a role in disc degeneration together with MMP-3 and MMP-11 enzymes with the same substrate properties.

As noted previously, disc degeneration starts in the early years of life. Epidemiological studies show that inheritance is the most important factor in disc degeneration. In a study conducted by Dong et al., it was stated that those who develop
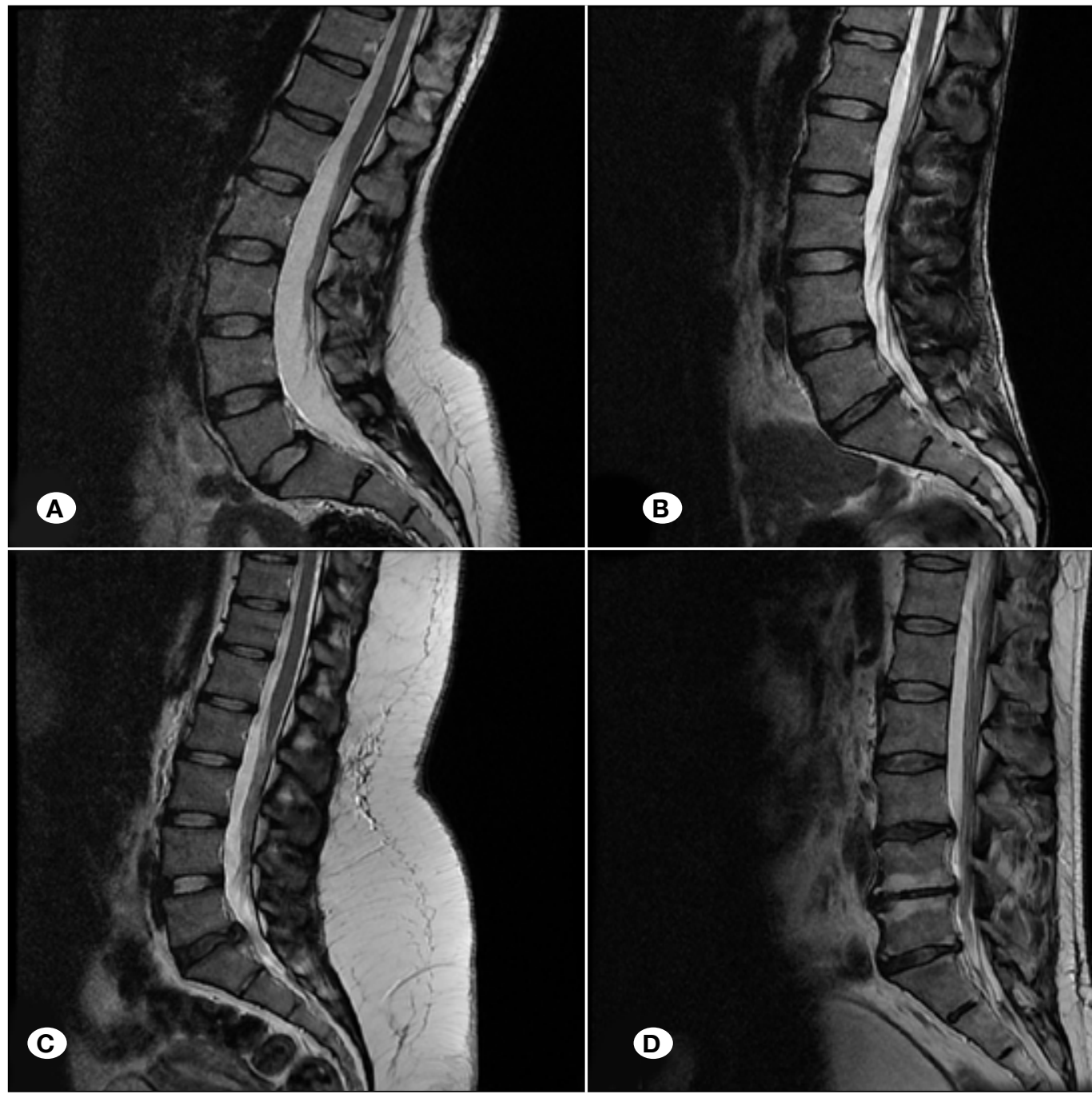

Figure 2: Sagittal section of T2 weighted MRI showed Schneiderman disc degeneration classification of patients. A) grade 0 - Normal (hyperintense signal),

B) grade I - slight reduction in signal,

C) grade II - global reduction in signal,

D) grade III - hypointense signal and disc space narrowing. 
disc degeneration in early years have more genetic tendency, and it was noted that MMP-2 $1306 \mathrm{C} / \mathrm{T}$ polymorphism may be a genetic risk factor associated with a tendency for lumbar disc disease in young adults (9).

In the current study, the role of MMP-11, which is another enzyme of stromelysin group, was investigated in disc degeneration by immunohistochemical staining of removed disc materials. There is no study related to this in the literature. However, an expression increase was shown in many diseases (7). In our study, while MMP-11 positivity was seen in $42.3 \%$ of disc materials taken during the operation, MMP-11 negativity was seen in $57.1 \%$. However, this rate was low when it was considered that other stromelysins with similar substrate property have important roles in disc degeneration. Although there is no data related with MMP11 in the literature, it was thought that MMP-11 may have a role in disc degeneration.

Lumbar disc herniation is more frequently seen in male and young-middle aged patients (9). The age limit was determined as 50 years old in our study by taking this into consideration and we divided the cases into two groups. In evaluations between the two groups, we found a statistically significant difference between MMP-11 staining degrees. This difference caused us to think that the MMP-11 enzyme may have a role in patients with disc degeneration in young-middle age. Prolongation of human life and ineffective treatment methods indicate that proposed new treatment choices that will prevent degeneration of intervertebral discs should be on the agenda. MMP continues to be important as a very strong mediator in the degeneration of intervertebral discs. Studies conducted in recent years focus on agents inhibiting synthesis and activity of $\operatorname{MMP}(3,12,18)$. Improvements in this regard are still in the experimental stage. Adverse effects of the substances investigated have meant they could not be used in humans until now. However, it may be possible to develop more specific therapeutic agents with a better understanding of macro-molecules of intervertebral discs, the effect of degeneration on pathogenesis and the regulation mechanism of matrix molecules by benefiting from immunohistochemistry and modern techniques.

In conclusion, it was shown in this study that MMP11 may play a role in the pathogenesis of intervertebral disc disease. However, disc degeneration is not a factor by itself. It is considered that it may be a risk factor genetically in youngmiddle aged patients.

This study showed the presence of MMP-11 enzyme in disc tissue with immunohistochemical staining in a small series. Wider series of immunohistochemical staining for MMP-11 and other MMPs, and simultaneous genetic studies, will illuminate the role of MMPs in the pathogenesis of disc degeneration and thus make it possible to develop more specific agents.

\section{REFERENCES}

1. Acaroglu ER, latridis JC, Setton LA, Foster RJ, Mow VC, Weidenbaum M: Degeneration and aging affect the tensile behavior of human lumbar anulus fibrosus. Spine (Phila $\mathrm{Pa}$ 1976) 20:2690-2701, 1995

2. Ahn SH, Cho YW, Ahn MW, Jang SH, Sohn YK, Kim HS: mRNA expression of cytokines and chemokines in herniated lumbar intervertebral discs. Spine (Phila Pa 1976) 27:911-917, 2002

3. Antoniou J, Steffen T, Nelson F, Winterbottom N, Hollander AP, Poole RA, Aebi M, Alini M: The human lumbar intervertebral disc: Evidence for changes in the biosynthesis and denaturation of the extracellular matrix with growth, maturation, ageing, and degeneration. J Clin Invest 98:996-1003, 1996

4. Barksby HE, Milner JM, Patterson AM, Peake NJ, Hui W, Robson T, Lakey R, Middleton J, Cawston TE, Richards CD, Rowan AD: Matrix metalloproteinase 10 promotion of collagenolysis via procollagenase activation: Implications for cartilage degradation in arthritis. Arthritis Rheum 54:32443253, 2006

5. Battie MC, Videman T, Gill K, Moneta GB, Nyman R, Kaprio J, Koskenvuo M: Smoking and lumbar intervertebral disc degeneration: An MRI study of identical twins. 1991 Volvo Award in clinical sciences. Spine (Phila Pa 1976) 16:10151021, 1991

6. Bijkerk C, Houwing-Duistermaat JJ, Valkenburg HA, Meulenbelt I, Hofman A, Breedveld FC, Pols HA, van Duijn $\mathrm{CM}$, Slagboom PE: Heritabilities of radiologic osteoarthritis in peripheral joints and of disc degeneration of the spine. Arthritis Rheum 42:1729-1735, 1999

7. Boulay A, Masson R, Chenard MP, El Fahime M, Cassard L, Bellocq JP, Sautes-Fridman C, Basset P, Rio MC: High cancer cell death in syngeneic tumors developed in host mice deficient for the stromelysin-3 matrix metalloproteinase. Cancer Res 61:2189-2193, 2001

8. Buckwalter JA: Aging and degeneration of the human intervertebral disc. Spine (Phila Pa 1976) 20:1307-1314, 1995

9. Dong DM, Yao M, Liu B, Sun CY, Jiang YQ, Wang YS: Association between the $-1306 \mathrm{C} / \mathrm{T}$ polymorphism of matrix metalloproteinase-2 gene and lumbar disc disease in Chinese young adults. Eur Spine J 16:1958-1961, 2007

10. Errington RJ, Puustjarvi K, White IR, Roberts S, Urban JP: Characterisation of cytoplasm-filled processes in cells of the intervertebral disc. J Anat 192 ( Pt 3):369-378, 1998

11. Goupille P, Jayson MI, Valat JP, Freemont AJ: Matrix metalloproteinases: The clue to intervertebral disc degeneration? Spine (Phila Pa 1976) 23:1612-1626, 1998

12. Guiot BH, Fessler RG: Molecular biology of degenerative disc disease. Neurosurgery 47:1034-1040, 2000

13. Handa $T$, Ishihara $H$, Ohshima $H$, Osada R, Tsuji $H$, Obata $\mathrm{K}$ : Effects of hydrostatic pressure on matrix synthesis and matrix metalloproteinase production in the human lumbar intervertebral disc. Spine (Phila Pa 1976) 22:1085-1091, 1997

14. Heliovaara M: Body height, obesity, and risk of herniated lumbar intervertebral disc. Spine (Phila Pa 1976) 12:469-472, 1987 
15. Heliovaara M, Knekt P, Aromaa A: Incidence and risk factors of herniated lumbar intervertebral disc or sciatica leading to hospitalization. J Chronic Dis 40:251-258, 1987

16. Kang JD, Stefanovic-Racic M, Mclntyre LA, Georgescu HI, Evans $\mathrm{CH}$ : Toward a biochemical understanding of human intervertebral disc degeneration and herniation. Contributions of nitric oxide, interleukins, prostaglandin E2, and matrix metalloproteinases. Spine (Phila Pa 1976) 22:1065-1073, 1997

17. Miller JA, Schmatz C, Schultz AB: Lumbar disc degeneration: Correlation with age, sex, and spine level in 600 autopsy specimens. Spine (Phila Pa 1976) 13:173-178, 1988

18. Nerlich AG, Schleicher ED, Boos N: 1997 Volvo Award winner in basic science studies. Immunohistologic markers for agerelated changes of human lumbar intervertebral discs. Spine (Phila Pa 1976) 22:2781-2795, 1997
19. Poiraudeau S, Monteiro I, Anract P, Blanchard O, Revel M, Corvol MT: Phenotypic characteristics of rabbit intervertebral disc cells. Comparison with cartilage cells from the same animals. Spine (Phila Pa 1976) 24:837-844, 1999

20. Rannou F, Corvol MT, Hudry C, Anract P, Dumontier MF, Tsagris L, Revel M, Poiraudeau S: Sensitivity of anulus fibrosus cells to interleukin 1 beta. Comparison with articular chondrocytes. Spine (Phila Pa 1976) 25:17-23, 2000

21. Richardson SM, Doyle $P$, Minogue BM, Gnanalingham K, Hoyland JA: Increased expression of matrix metalloproteinase-10, nerve growth factor and substance $P$ in the painful degenerate intervertebral disc. Arthritis Res Ther 11:R126, 2009

22. Schneiderman G, Flannigan B, Kingston S, Thomas J, Dillin WH, Watkins RG: Magnetic resonance imaging in the diagnosis of disc degeneration: Correlation with discography. Spine (Phila Pa 1976) 12:276-281, 1987 